\title{
Avaliação do risco coronariano e sua relação com as ações de saúde em hipertensos
}

\author{
Evaluation of coronary risk and its relationship to health actions in hypertensive patients \\ Evaluación del riesgo coronario y su relación con las acciones de salud en hipertensos
}

\author{
Viviane Rassele Silva', Maria del Carmen Bisi Molina", Nagela Valadão Cade"l \\ I Prefeitura Municipal de Vitória, Secretaria Municipal de Saúde. Vitória-ES, Brasil. \\ "Universidade Federal do Espírito Santo, Programa de Pós-Graduação em Saúde Coletiva. Vitória-ES, Brasil.
}

Submissão: 15-11-2012 Aprovação: 25-07-2014

\section{RESUMO}

Avaliou-se o risco para desenvolvimento de eventos coronarianos agudos de acordo com os critérios de Framingham e com as ações de saúde realizadas em hipertensos de uma Unidade de Saúde da Família de Vitória-ES. Estudo observacional, de corte transversal, envolvendo 330 hipertensos. A amostra considerou prevalência do agravo de $50 \%$. Os dados foram coletados dos prontuários e as variáveis constituíram o risco coronariano, a pressão arterial, o número de consultas, de atividades educativas e de medicamentos prescritos. Foi utilizada a ANOVA para comparar as variáveis e o teste t pareado para comparação da pressão no período estudado, com nível de significância de 5\%. Respectivamente, $115(34,8 \%)$ sujeitos apresentaram baixo risco de infarto ou morte por doença coronariana nos próximos 10 anos; 67 (20,4\%) apresentaram médio risco; e 148 (44,8\%) apresentaram alto risco. Somente o quantitativo de medicamentos prescritos mostrou relação significante com o risco coronariano elevado. Descritores: Avaliação; Grupos de Risco; Hipertensão; Programa Saúde da Família; Serviços de Saúde.

\begin{abstract}
The risk of developing acute coronary events was evaluated according to Framingham criteria and health actions performed to hypertensive patients at a Health Unit Family of Vitória-ES. This is a observational, cross-sectional study, involving 330 hypertensive. The sample considered a $50 \%$ prevalence of the disease. Data were collected from medical records and the variables were the coronary risk, blood pressure, the number of visits, educational activities and prescribed drugs. ANOVA was used to compare variables and paired t-test for comparison of pressure during the study period, with significance level of $5 \%$. Respectively, 115 (34.8\%) subjects had low risk for myocardial infarction or death from coronary heart disease in the next 10 years; $67(20.4 \%)$ had average risk; and $148(44.8 \%)$ had high-risk. Only the amount of prescribed medications showed significant relationship with high coronary risk.
\end{abstract}

Key words: Assessment; Risk Groups; Hypertension; Family Health Program; Health Services.

\section{RESUMEN}

Se evaluó el riesgo de desarrollar eventos coronarios agudos según criterios de Framingham y acciones de salud realizadas en hipertensos de una Unidad de Salud de la Familia de Vitória-ES. Estudio observacional, transversal, envolviendo la participación de 330 hipertensos. La muestra considero la prevalencia del agravo en 50\%. Los datos fueron recogidos de las historias clínicas y las variables fueron el riesgo coronario, presión arterial, el número de visitas, actividades educativas y los medicamentos recetados. Fue utilizada ANOVA para comparar las variables y el teste t para la comparación de la presión durante el período de estudio, con un nivel de significación del 5\%. Respectivamente, 115 (34,8\%) sujetos tenían bajo riesgo de infarto de miocardio o muerte por enfermedad coronaria en los próximos 10 años; 67 (20,4\%) tenían un riesgo promedio; y 148 (44,8\%) tenían alto riesgo. Sólo la cantidad de medicamentos prescritos mostraron asociación significativa con el riesgo coronario alto.

Palabras clave: Evaluación; Grupos de Riesgo; Hipertensión; Programa de Salud Familiar; Servicios de Salud. 


\section{INTRODUÇÃO}

No âmbito mundial, inclusive no Brasil, as doenças cardiovasculares (DCV) constituem a primeira causa de morbimortalidade em adultos, e cada vez mais pessoas morrem anualmente dessas doenças, em relação a qualquer outra causa ${ }^{(1-2)}$.

Uma dessas doenças é a hipertensão arterial sistêmica (HAS) que, por sua vez, multiplica o risco para desenvolvimento de outras DCV. Nesse sentido, é um importante fator de risco a ser considerado na morbimortalidade cardiovascular ${ }^{(3,4)}$.

Na assistência ao portador de HAS, as ações de saúde devem contemplar a abordagem para redução dos fatores de risco para as DCV através de terapêutica medicamentosa e mudanças no estilo de vida. O sucesso do controle desses fatores depende da adesão do paciente e, para isso, a equipe de saúde deve utilizar a educação em saúde como ferramenta principal, além das consultas individuais(4-5).

Todas essas intervenções devem ser realizadas no primeiro nível de atenção à saúde, especificamente na Estratégia de Saúde da Família (ESF) que, por sua conformação e processo de trabalho, pressupõe vínculo com a comunidade e usuários, e melhores condições para controle dos fatores de risco para as doenças cardiovasculares, pela natureza multidisciplinar dos atendimentos ${ }^{(5-6)}$.

Com relação ao atendimento clínico, as diretrizes e os protocolos para tratamento da HAS recomendam a avaliação do risco cardiovascular em hipertensos e uma forma de classificá-lo é a utilização de algoritmos, sendo o modelo de Framingham recomendado pelas diretrizes brasileiras ${ }^{(5,7)}$.

O estudo de Framingham, iniciado em 1948, teve como objetivo investigar os fatores de risco para as doenças do coração, pois foi uma época de transição epidemiológica em que a mortalidade e a incidência da DCV aumentavam progressivamente e pouco se conhecia sobre seus fatores de risco e fisiopatologia. Foi um estudo de longa duração, que permitiu definir e estratificar o risco cardiovascular como a probabilidade de ocorrer um evento cardiovascular em 10 anos e para a estratificação foram utilizadas variáveis clínicas e laboratoriais, utilizadas na prática clínica.

Considerando que os hipertensos que participam de forma mais frequente das ações de saúde propostas pela Estratégia de Saúde da Família têm a possibilidade de reduzir os fatores envolvidos no risco cardiovascular, este estudo objetivou avaliar o risco para desenvolvimento de eventos coronarianos agudos - infarto agudo do miocárdio ou morte por doença coronariana nos próximos 10 anos - de acordo com os critérios de Framingham, e relacionar este risco com as ações de saúde realizadas em hipertensos atendidos em uma Unidade de Saúde da Família, em Vitória-ES.

\section{METODOLOGIA}

Trata-se de estudo observacional, de corte transversal realizado em uma Unidade de Saúde da Família (USF) do município de Vitória, ES com hipertensos cadastrados no Sistema de Informação da Atenção Básica (SIAB) da USF. Para o cálculo amostral foram considerados os parâmetros para populações finitas, a prevalência do agravo de 50\%, população de 2676 sujeitos, conforme dados do SIAB de setembro de 2009, nível de significância de 95\% e erro amostral de 5\%. A amostra foi constituída por um mínimo de 330 hipertensos, aleatorizados por sorteio sistemático.

Como critérios de inclusão foram considerados: ser hipertenso, ter idade igual ou maior a 18 anos, ter registro no prontuário de pelo menos uma medida de pressão arterial (PA) e um resultado de colesterol total (CT), HDL colesterol (HDL-c) e de glicemia de jejum em um período de 18 meses, de juIho de 2008 a dezembro de 2009, resultados utilizados para cálculo do risco coronariano. Como critério de exclusão foi considerado o indivíduo com diagnóstico de hipertensão secundária, segundo registro no prontuário.

Os dados foram coletados diretamente dos prontuários dos hipertensos e a pesquisa foi aprovada pelo Comitê de Ética e Pesquisa com Seres Humanos da Universidade Federal do Espírito Santo, sob o número 011/10.

Foram estudados o risco coronariano e as variáveis relacionadas com as ações de saúde - pressão arterial, número de consultas, de reuniões educativas e de medicamentos prescritos. No que concerne às ações de saúde, foram registrados todos os atendimentos individuais realizados pelos diversos profissionais, bem como a participação em ações educativas desenvolvidas em grupo no período de julho de 2008 a dezembro de 2009. Também foram computadas as visitas domiciliares nesse período e a medicação prescrita na última consulta (médica ou de enfermagem).

Para o cálculo do risco coronariano optou-se por usar a escala proposta pela Sociedade Brasileira de Cardiologia na formulação da IV Diretriz Brasileira sobre Dislipidemias e Prevenção da Aterosclerose que classifica o risco coronariano em baixo probabilidade menor de $10 \%$ de ter evento coronariano agudo; médio - probabilidade entre 10 e $20 \%$; e alto - probabilidade maior que $20 \%$. O algoritmo de Framingham utilizado neste estudo emprega os valores de CT, de HDL-c, de pressão arterial sistólica e a presença de diabetes e de tabagismo ${ }^{(7)}$.

Para a avaliação do valor da pressão arterial, do CT e do HDL-c foram utilizadas as últimas medidas registradas no prontuário nos dezoito meses do estudo. Como critério de tabagismo foi convencionado o uso de cigarro diariamente, independente da quantidade; foram considerados como diabéticos aqueles que tinham prescrição médica de hipoglicemiantes orais ou de insulina ou valores de glicemia em jejum alterados $^{(7)}$. Também foram registrados os últimos valores do LDL-c por constituir fração do CT.

As variáveis quantitativas (pressão arterial, número de consultas e de reuniões educativas) foram analisadas através de média e desvio padrão e comparadas pela ANOVA. O teste $t$ pareado foi utilizado para comparação da pressão arterial no período estudado. Foi considerada uma associação estatística significante quando $\mathrm{p}<0,05$. Para análise estatística foi utilizada a versão 15.0 do pacote estatístico SPSS.

\section{RESULTADOS}

Dos 696 prontuários acessados, houve perda de 366 por não atenderem aos critérios de inclusão do estudo. Desses 366, 256 
sujeitos não possuíam registro de exames laboratoriais nos dezoito meses do estudo; 63 prontuários não foram encontrados no arquivo da Unidade de Saúde; 47 sujeitos não tinham registro de consulta nos dezoito meses, sendo que três deles eram acompanhados por profissionais do plano de saúde, e um sujeito não mais residia na área de abrangência da Unidade de Saúde da Família. Dessa forma, foi necessário acessar mais do que o dobro de prontuários para se chegar à amostra de 330 hipertensos.

Dos 330 sujeitos estudados, 199 (60,3\%) não tinham registro do uso de tabaco no prontuário, sendo necessário buscar essa informação com os agentes comunitários de saúde da Unidade.

Sobre a variável pressão arterial, a média da pressão sistólica (PAS) foi de 138 ( \pm 20 ) e da pressão diastólica (PAD) foi de 85 ( \pm 13$) \mathrm{mmHg}$; porém, de acordo com o valor da mediana (140x80), a metade dos sujeitos apresentou PAS acima de 140 mmHg. Além disso, 201 (60,9\%) sujeitos apresentavam pressão arterial elevada. Foram utilizados neste estudo os termos pressão arterial elevada, segundo a orientação da VI Diretriz Brasileira de Hipertensão Arterial, que considera satisfatória a redução da pressão arterial para valores abaixo de $140 \times 90$ $\mathrm{mmHg}$ nos pacientes hipertensos sem complicações. Por outro lado, para aqueles de elevado risco cardiovascular, com diabetes e com nefropatia, deve-se considerar como meta do tratamento valor inferior a $130 \times 80 \mathrm{mmHg}^{(4)}$.

Também foi avaliado o comportamento da pressão ao longo de 12 meses, visto que alguns epidemiologistas sugerem que a PA é um parâmetro quantitativo e não deve ser descrita ou categorizada de acordo com um sistema de base binária (elevada ou não elevada), pois, quanto mais alta a pressão

Tabela 1 - Pressão arterial em três momentos em 110 hipertensos

\begin{tabular}{rrrrrrrrrr}
\hline Pressão & \multicolumn{3}{r}{ Inicial } & \multicolumn{3}{c}{6 meses } & \multicolumn{3}{c}{ 12 meses } \\
\hline & Média & DP & Md & Média & DP & Md & Média & DP & Md \\
\hline PAS & 136,9 & 21,2 & 130 & $137,4^{\text {NS }}$ & 21,5 & 135 & $136,4^{\text {NS }}$ & 24,8 & 130 \\
PAD & 85,5 & 13,5 & 80 & $84,3^{\text {NS }}$ & 12,1 & 80 & $85,7^{\text {NS }}$ & 17,7 & 80 \\
\hline
\end{tabular}

Teste t pareado entre inicial x 6 meses e inicial x 12 meses.

NS - não significante

Tabela 2 - Número de consultas e reuniões educativas frequentadas pelos hipertensos

\begin{tabular}{lccccc}
\hline Variável & Média & D. Padrão & Mínimo & Máximo & Mediana \\
\hline Total de consultas & 7,1 & 3,2 & 1,0 & 18,0 & 7,0 \\
$\quad$ Médicos & 5,6 & 2,6 & 0,0 & 14,0 & 5,0 \\
Enfermeiros & 1,3 & 1,4 & 0,0 & 7,0 & 1,0 \\
Outros profissionais & 0,2 & 0,5 & 0,0 & 4,0 & 0,0 \\
Faltas & 0,6 & 0,8 & 0,0 & 4,0 & 0,0 \\
Total de reuniões & 1,6 & 1,3 & 0,0 & 5,0 & 1,0 \\
$\quad$ Médicos & 2,0 & 1,1 & 0,0 & 5,0 & 2,0 \\
Enfermeiros & 0,7 & 0,8 & 0,0 & 4,0 & 1,0 \\
$\quad$ Outros profissionais & 2,1 & 1,1 & 0,0 & 6,0 & 2,0
\end{tabular}

arterial, maiores serão as consequências no organismo.

Dos 330 sujeitos, 110 apresentaram registro em prontuário de pelo menos dois valores de PA com intervalo de seis meses durante os 18 meses do estudo e 28 sujeitos tinham três registros de pressão com intervalo de seis meses, no período analisado. Foi observado que as médias apresentaram pouca variação e não houve diferença estatística entre elas (Tabela 1).

Com relação às ações realizadas pelos profissionais de saúde da Unidade, os hipertensos do estudo frequentaram uma média de 7,1 $( \pm 3,2)$ consultas nos dezoito meses, o que corresponde a uma consulta a cada dois meses e meio. As faltas nas consultas foram relativamente baixas, menor que uma falta nos dezoitos meses (Tabela 2). Destaca-se que só foram computadas as consultas médicas e de enfermagem que, de alguma forma, estavam relacionadas com o tratamento da hipertensão.

Dessas consultas, a maioria, 5,6 $( \pm 2,6)$, foi realizada pelo médico, seguido pelo enfermeiro, com $1,3( \pm 1,4)$. O número de atendimento realizado por outros profissionais (psicólogos, educador físico, assistente social e farmacêutico) foi baixo nos 18 meses do estudo, 0,2 $( \pm 0,5)$ (Tabela 2 ).

Sobre as reuniões educativas realizadas em grupo, algumas foram realizadas por mais de um profissional e, nesse caso, todos eles foram considerados neste estudo. Na Tabela 2 observa-se que os sujeitos participaram em média de $1,6( \pm 1,3)$ reuniões no período estudado, e destaca-se que um pouco mais de um terço da amostra não tinha registro em prontuário quanto à participação de algum tipo de reunião educativa. Dos profissionais da equipe, houve a participação em maior frequência do médico e do enfermeiro. Encontrou-se registro da participação de outros profissionais, como o farmacêutico, psicólogo, educador físico e auxiliar de enfermagem nas reuniões educativas em menor quantidade, média 2,1 $( \pm 1,1)$ (Tabela 2).

Também foi observado durante análise dos prontuários que a atividade educativa foi organizada diferentemente nas equipes de saúde, e não foram encontrados registros desse tipo de atividade nos prontuários de quatro equipes de saúde da família dentre as sete equipes que trabalham na USF.

Além dessas ações, consultas e reuniões, foram registradas as visitas domiciliares realizadas, em sua maioria, por auxiliares de enfermagem e enfermeiros, assim como as visitas médicas. Ao todo $64(19,4 \%)$ hipertensos receberam visitas no domicílio, entre 4 e 17 visitas no período de 18 meses.

Com relação às medicações em uso, dos 330 prontuários analisados, 12 (3,6\%) sujeitos não utilizavam nenhuma medicação, pois o controle da pressão estava sendo realizado somente com as medidas higienodietéticas. Daqueles que faziam uso de medicação, 175 (53\%) sujeitos possuíam registro de uma a duas classes de medicação e 143 (43,3\%) utilizavam três ou mais medicamentos diferentes.

Quanto ao esquema terapêutico foram encontradas 12 classes de medicações prescritas nos prontuários, quais sejam: diurético tiazídico 
(hidroclorotiazida), diurético de alça (furosemida), diurético poupador de potássio (espironolactona), inibidores da enzima de conversão da angiotensina I - ECA (captopril e enalapril), betabloqueador (atenolol e propranolol), bloqueador da angiotensina I (losartana potássica), bloqueador de canais de cálcio (anlodipina e nifedipina), inibidor adrenérgico (metildopa), hipoglicemiante oral (glibenclamida e metformina), hipoglicemiante parenteral (Insulina), antiplaquetário (AAS), hipolipemiante - estatinas (sinvastatina), medicamentos esses dispensados pela rede municipal de saúde dos municípios.

Tabela 3 - Pressão arterial em três momentos nos 148 hipertensos de alto risco

\begin{tabular}{|c|c|c|c|c|c|c|c|c|c|}
\hline \multirow{3}{*}{ Pressão } & \multicolumn{8}{|c|}{ Tempo } & \\
\hline & \multicolumn{3}{|c|}{ Inicial } & \multicolumn{3}{|c|}{6 meses } & \multicolumn{3}{|c|}{12 meses } \\
\hline & Média & DP & Md & Média & DP & Md & Média & DP & Md \\
\hline PAS & 141,6 & 20,1 & 140 & $144,7^{\mathrm{NS}}$ & 21,2 & 140 & $139,2^{\mathrm{NS}}$ & 11,6 & 130 \\
\hline PAD & 85,1 & 14,5 & 80 & $86,3^{\mathrm{NS}}$ & 11,3 & 90 & $82,5^{\text {NS }}$ & 12,2 & 80 \\
\hline
\end{tabular}

Teste $t$ pareado entre inicial x 6 meses e inicial $\times 12$ meses NS - não significante

Tabela 4 - Relação entre as consultas e reuniões educativas realizadas pelos profissionais de saúde e o risco de infarto ou morte por doença coronariana no período de 10 anos, segundo critério de Framingham

\begin{tabular}{|c|c|c|c|c|c|c|c|}
\hline \multirow{3}{*}{ Variável } & \multicolumn{7}{|c|}{ Risco Coronariano } \\
\hline & \multicolumn{2}{|l|}{ Baixo } & \multicolumn{2}{|l|}{ Médio } & \multicolumn{2}{|l|}{ Alto } & \multirow[t]{2}{*}{ p-valor } \\
\hline & Média & DP & Média & DP & Média & DP & \\
\hline Total consultas & 7,0 & 3,1 & 7,2 & 3,0 & 7,1 & 3,4 & 0,911 \\
\hline Médicos & 5,3 & 2,4 & 6,0 & 2,8 & 5,6 & 2,6 & 0,260 \\
\hline Enfermeiros & 1,5 & 1,4 & 1,0 & 1,2 & 1,3 & 1,4 & 0,058 \\
\hline Outros profissionais & 0,2 & 0,6 & 0,2 & 0,5 & 0,2 & 0,5 & 0,823 \\
\hline Total de reuniões & 1,7 & 1,4 & 1,6 & 1,2 & 1,5 & 1,3 & 0,325 \\
\hline Médicos & 2,1 & 1,1 & 1,9 & 1,1 & 1,9 & 1,1 & 0,479 \\
\hline Enfermeiros & 0,9 & 0,9 & 0,7 & 0,8 & 0,6 & 0,8 & 0,065 \\
\hline Outros profissionais & 2,3 & 1,1 & 2,0 & 1,0 & 2,0 & 1,1 & 0,196 \\
\hline
\end{tabular}

Tabela 5 - Relação entre o número de medicações utilizadas e o risco de infarto ou morte por doença coronariana no período de 10 anos, segundo Framingham

\begin{tabular}{lcccccccc}
\hline & \multicolumn{8}{c}{ Risco Cardiovascular } \\
Variável & \multicolumn{2}{c}{ Baixo } & \multicolumn{2}{c}{ Médio } & \multicolumn{2}{c}{ Alto } & \multirow{2}{*}{ p-valor } \\
\cline { 2 - 6 } & $\mathbf{n}$ & $\mathbf{\%}$ & $\mathbf{n}$ & $\mathbf{\%}$ & $\mathbf{n}$ & $\mathbf{\%}$ & \\
\hline Medicações & & & & & & & \\
Até duas & 88 & 47,1 & 42 & 22,5 & 57 & 30,5 & 0,000 \\
Três ou mais & 27 & 18,9 & 25 & 17,5 & 91 & 63,6 & \\
\hline
\end{tabular}

No que tange ao risco coronariano global, a média encontrada foi de $11,5 \%( \pm 9,2)$ e a mediana de 8,0 , sendo que 115 $(34,8 \%)$ sujeitos apresentaram risco baixo, 67 (20,4\%) risco médio e $148(44,8 \%)$ risco alto de desenvolver eventos coronarianos nos próximos 10 anos. Destaca-se que a média não considerou os sujeitos portadores de diabetes da amostra 103 $(31,2 \%)$ e outros agravos, pois são condições que, conforme o algoritmo utilizado, classificam o indivíduo como risco elevado.

Dos 148 (44,8\%) indivíduos categorizados como alto risco, $102(68,9 \%)$ apresentaram LDL colesterol acima de $100 \mathrm{mg} /$ dl, valor meta de tratamento para esses indivíduos de acordo com a Sociedade Brasileira de Cardiologia (2007). Desses indivíduos com LDL elevado apenas $12(11,7 \%)$ tinham o registro do uso da sinvastatina. Dos 67 (20,4\%) indivíduos com médio risco, 38 (56,7\%) possuíam LDL acima de $130 \mathrm{mg} /$ $\mathrm{dl}$, valor meta para essa categoria de risco, e apenas $5(13,1 \%)$ tinham prescrição de sinvastatina.

Ainda com relação aos $148(44,8 \%)$ sujeitos com alto risco coronariano, 48 (32,4\%) possuíam pelo menos duas medidas de PA no intervalo de 6 meses e $12(8,1 \%)$ sujeitos tinham três registros de PA em dezoito meses (Tabela 3 ).

Em análise do comportamento da PA nos indivíduos com alto risco observa-se que não houve diferença estatística entre as médias ao longo do tempo, sendo que a PAS manteve-se pouco elevada nos dois momentos iniciais e aos doze meses, as médias e medianas reduziram de forma não significante. (Tabela 3).

Na relação do risco coronariano com as ações de saúde (consultas e atividades educativas), os dados mostraram que não houve associação estatisticamente significante. Em análise descritiva, o número de consultas e de reuniões educativas realizadas por enfermeiros é menor naqueles hipertensos de risco coronariano alto, em relação aqueles de baixo risco (Tabela 4).

Houve associação entre a quantidade de classes de medicação consumida com o risco coronariano. Os indivíduos com maior risco usam, em sua maioria, três ou mais classes de medicamentos, enquanto a maior parte dos indivíduos de baixo risco faz uso de uma ou duas medicações diferentes $(p$ $0,000)$, mostrando que aqueles de maior risco fazem uso de mais medicamentos (Tabela 5).

\section{DISCUSSÃO}

Grande parte dos prontuários foi descartada por não possuir registro de consulta, de exames laboratoriais e informações sobre o hábito de fumar no período do estudo e, por vezes, o prontuário não foi encontrado, o que além de dificultar a coleta de dados, entendemos ser uma limitação do estudo, à medida que pode introduzir um viés de seleção da amostra. 
Tais situações podem remeter a três questões: a) o não registro dos resultados de exames e da consulta; b) o usuário não retorna para mostrar os exames aos profissionais e dar continuidade ao tratamento; c) falha na organização do serviço no que diz respeito à manutenção do arquivo e extravio dos prontuários; e d) falta de padronização da conduta, pois a VI Diretriz de Hipertensão sugere que o hipertenso deva consultar no mínimo a cada seis meses quando não apresenta complicações. Não foram encontrados nos documentos oficiais sobre o tratamento da hipertensão informações sobre a periodicidade de solicitação de exames bioquímicos, mas os consensos determinam avaliação laboratorial de rotina, com periodicidade no mínimo anual(4).

A ocorrência de quaisquer dessas possibilidades sugere problemas na estruturação do processo de trabalho do serviço de saúde e prejudica o acompanhamento de dados clínicos, como a pressão arterial, os dados laboratoriais e o tratamento, se distanciando das proposições do Ministério da Saúde, o que implica em discutível qualidade na continuidade do cuidado oferecido ${ }^{(4-5)}$.

No que se refere aos dados laboratoriais, a IV Diretriz de Dislipidemia estabelece valores de referência para os componentes do perfil lipídico de acordo com a classificação de risco coronariano. Com relação ao LDL-C, para os indivíduos de baixo risco a meta a ser alcançada é abaixo de $160 \mathrm{mg} / \mathrm{dl}$; para aqueles que possuem risco médio a meta diminui para $130 \mathrm{mg} / \mathrm{dl}$ e para os de alto risco deve-se ter LDL-c menor que $100 \mathrm{mg} / \mathrm{dl}^{(7)}$.

Sob essa perspectiva, embora o valor de LDL estivesse acima da meta para aqueles do médio e alto risco para eventos coronarianos, apenas $11,7 \%$ dos hipertensos tinham algum tipo de estatina prescrita. Essa classe de medicamento tem a propriedade de reduzir o LDL-c de $15 \%$ a $55 \%$ em adultos; diminuir o TG de $7 \%$ a $28 \%$ e elevar o HDL-c de $2 \%$ a $10 \%$ e dessa forma, são capazes de reduzir a mortalidade cardiovascular, a incidência de eventos isquêmicos coronários agudos, a necessidade de revascularização do miocárdio e a ocorrência do acidente vascular encefálico ${ }^{(7)}$.

Ao ser avaliado o comportamento da pressão arterial ao longo de 12 meses em 110 sujeitos da amostra que tinham pelo menos duas medidas nesse intervalo de tempo, foi observado que as médias apresentaram pouca variação e não tiveram diferença estatística entre elas, sugerindo que a PA desses 110 hipertensos se manteve constante ao longo do tempo e com valores abaixo de $140 \times 90 \mathrm{mmHg}$ (Tabela 1 ).

No entanto, os últimos valores registrados em prontuário revelaram percentual de 201 (60,9\%) indivíduos com PA igual ou acima de $140 \times 90 \mathrm{mmHg}$, dados esses mais elevados em relação a outros estudos também realizados com hipertensos em acompanhamento em Unidade de Saúde da Família ${ }^{(8-9)}$.

Esses valores de pressão arterial elevados, em hipertensos cadastrados e monitorados por equipes de saúde, atendidos em estabelecimento que fornece praticamente todos os medicamentos necessários para o controle da PA, nos leva a inferir que os usuários podem não utilizar a medicação conforme a prescrição, ou seja, apresentam baixa adesão ao tratamento medicamentoso. Apesar de não ter sido o foco deste estudo a literatura reforça essa possibilidade, pois pesquisa realizada em um ambulatório-escola de São Paulo identificou que 85,76\% dos hipertensos não aderiram ao tratamento medicamentoso e $85,29 \%$ ao tratamento não medicamentoso, mesmo apresentando em sua maioria $(61,76 \%)$ assiduidade às consultas ${ }^{(10)}$. Outros estudos também evidenciam a baixa adesão ao tratamento em hipertensos atendidos em unidade básica de saúde. Em Maringá-PR, 64\% e em Terezina-PI, 77,5\% dos hipertensos não eram aderentes ao tratamento ${ }^{(11-12)}$. Em perspectiva qualitativa, estudo no Paraná mostra o relato dos profissionais de saúde quanto as dificuldades encontradas para efetivarem as estratégias de adesão ao tratamento em hipertensos ${ }^{(13)}$. Nesse sentido, a adesão ao tratamento é uma questão importante a ser avaliada pelos profissionais de saúde.

A pressão arterial é aferida na USF em questão uma única vez antes da consulta, seja médica ou de enfermagem, nos indivíduos sentados e após repouso de pelo menos 10 minutos com utilização de aparelho oscilométrico (coluna de mercúrio e aneróide) que recebem manutenção preventiva uma vez por mês. Porém, não é uma prática do serviço realizar treinamentos e procedimentos de controle de qualidade quanto à técnica de verificação da pressão e, dessa forma, deve ser considerada a possibilidade de que as medidas apresentadas podem ter um viés de verificação.

A média de consultas revelou um número de atendimentos médico a cada 3,2 meses e anual para o enfermeiro. Os documentos e protocolos oficiais que discorrem sobre o monitoramento e tratamento da hipertensão sugerem que a avaliação clínica do médico deve ocorrer pelo menos duas vezes ao ano em indivíduos com a PA controlada, sem sinais de lesões em órgãos-alvo e sem comorbidades. Já para os indivíduos que, mesmo apresentando controle das cifras pressóricas e da glicemia, tenham lesões em órgãos-alvo ou comorbidades, as consultas devem ser trimestrais ${ }^{(6,9)}$. Nessa lógica, se todos os sujeitos estivessem com a PA controlada, poderíamos dizer que o número de atendimento médico neste estudo foi satisfatório; porém não é possível fazer essa asserção devido aos dados referentes à pressão arterial apresentados.

Já para as consultas de outros profissionais a média foi baixa, principalmente pelo fato de que, nesse quesito, encontravam-se enfermeiro e outros profissionais (educador físico, psicólogo, farmacêutico e outros) que devem compor a equipe multiprofissional de atendimento ao hipertenso, tanto com atribuições no atendimento individual, como também nas atividades coletivas para a educação em saúde, pois a abordagem multiprofissional é essencial na atenção ao portador de hipertensão, por ser uma doença multifatorial que envolve cuidados para vários objetivos, e assim, com olhares de vários profissionais, seu tratamento tende a ser mais efetivo ${ }^{(4,7)}$.

Dentre as intervenções não medicamentosas da hipertensão arterial - redução do peso, do consumo de sal, aumento da ingestão de legumes, frutas e fibras e redução da gordura, atividade física, controle do estresse psicossocial dentre outros, a abordagem multiprofissional apresenta o mais alto grau de consenso e evidência científica quanto ao seu uso na prática clínica, recomendação essa baseada em múltiplos ensaios clínicos controlados ${ }^{(4,7)}$. 
O pequeno número de atividades educativas encontrado neste estudo pode ser decorrente de um baixo registro dessas atividades. No entanto, entende-se que esse registro é fundamental na organização dos serviços de Estratégia de Saúde da Família visto que a educação em saúde é uma das atribuições da equipe que atende o hipertenso e um componente importante no processo de aprendizagem, necessário no transcorrer da doença crônica, contribuindo para a ampliação de habilidades e comportamentos, bem como melhor adaptação à doença.

Por outro lado, estudo de revisão sobre as práticas de cuidado de enfermagem ao hipertenso nas produções científicas dos últimos dez anos identificou o predomínio da Consulta de Enfermagem e de forma assistemática, individualizada e centrada no modelo médico hegemônico, em vez de uma prática ancorada em trabalho educativo e de cunho coletivo com possibilidades de proporcionar melhor adaptação a cronicidade da doença e maior adesão ao tratamento ${ }^{(14)}$.

Ao ver a distribuição das atividades educativas e das consultas de acordo com o risco coronariano (baixo, médio ou alto), observou-se na Tabela 4 que mantiveram frequências baixas e semelhantes, sem significância estatística, independente da categoria de risco coronariano e esse resultado demonstra que os profissionais não têm discriminado os riscos individuais desses hipertensos a ponto de intensificarem as ações de saúde para aqueles que apresentam maiores condições de risco.

Por fim, pelos registros encontrados, parece que o atendimento do hipertenso na USF estudada está centrado no profissional médico, diminuindo as possibilidades de uma abordagem para além do tratamento medicamentoso. Nesse contexto, também é importante a inserção de outros profissionais no atendimento individual para orientação do uso correto da medicação, principalmente o enfermeiro e o farmacêutico e no atendimento coletivo, com a participação da equipe de apoio, pois o tratamento do hipertenso não deve envolver somente a redução da pressão arterial. O controle dos demais fatores de risco associados deve ser buscado.

Quando se relacionou a medicação com o risco para infarto ou morte por doença coronariana ficou evidente que os indivíduos de alto risco utilizam mais medicações do que os de baixo e médio risco, o que é compreensível na medida em que grande parte dos indivíduos com alto risco possui mais fatores determinantes do agravo, como diabetes, acidente vascular encefálico, dislipidemia e outros, demandando outras drogas além dos anti-hipertensivos.

O tratamento medicamentoso é necessário à maioria dos pacientes hipertensos e apresenta como objetivo principal a redução da morbidade e da mortalidade cardiovascular. Dessa forma, os anti-hipertensivos devem não só reduzir a pressão arterial, mas também os eventos cardiovasculares fatais e não-fatais ${ }^{(4)}$.

\section{CONCLUSÃO}

Neste estudo ao avaliar o risco para desenvolvimento de eventos coronarianos agudos de acordo com os critérios de Framingham e relacioná-lo com as ações de saúde realizadas na população hipertensa atendida em uma Unidade de Saúde da Família concluiu-se que não houve diferença de conduta dos profissionais de saúde ao atenderem o hipertenso de acordo com o grau de risco. Mesmo que não fosse utilizada nenhuma forma de classificação de risco, mais da metade dos sujeitos estavam com a pressão arterial e LDL colesterol elevados e se forem consideradas as metas para o tratamento dessas condições, a discrepância entre o valor encontrado e o valor ideal, aumenta.

Ainda, pelos registros, houve baixa atuação de outros profissionais não médicos no atendimento do hipertenso o que sugere a necessidade de maior participação da equipe multiprofissional.

Evidentemente, não se pretendeu uma análise do desempenho do serviço de saúde, no entanto, a análise dos dados permitiu discussões sobre o cuidado oferecido e a organização de uma Unidade de Saúde da Família, considerando que sua implementação pressupõe, dentre outros, uma visão ampliada do conceito de saúde, de ações de vigilância e reorientação do trabalho com ênfase nos grupos populacionais em situação de risco, como é o caso dos hipertensos.

Os resultados observados neste estudo podem ser trabalhados pela Estratégia de Saúde da Família, considerando as características desse tipo de serviço, pois: a) apresenta equipes de saúde da família responsáveis por um contingente restrito de residentes de determinado bairro, e com isso possuem informações sobre as condições de saúde dos usuários sob sua responsabilidade; b) a essas equipes estão integrados outros profissionais de apoio da ESF como, psicólogo, assistente social, educador físico, farmacêutico, com maiores possibilidades de tratamento e acompanhamento do usuário por equipe multiprofissional; c) tem a sistemática de busca de faltosos e visita domiciliar para aqueles que necessitam, e d) utiliza como ferramenta principal a educação em saúde, seja ela desenvolvida individualmente durante a consulta ou em grupos.

A utilização da classificação de risco global em hipertensos ao determinar o risco pode contribuir para o planejamento das ações de saúde de forma tal que a equipe de saúde possa dispensar uma atenção diferenciada e mais qualificada ao portador de hipertensão.

\section{REFERÊNCIAS}

1. Ministério da Saúde. Informações de Saúde. Mortalidade [Internet] Brasília (DF): DATASUS; [s.d] [acesso em 05 de julho de 2014]. Disponível em: http://datasus.saude.gov. br/informacoes-de-saude

2. World Health Organization. Cardiovascular diseases [Internet]
Genebra (CH): WHO; [s.d] [cited 2014 June 31]. Available from: http://www.who.int/topics/cardiovascular_diseases/en

3. Pereira JC, Barreto SM, Passos VMA. Perfil de risco cardiovascular e autoavaliação de saúde no Brasil: um estudo de base populacional. Rev Panam Salud Pública [Internet]. 
2009 [acesso em 05 de julho de 2014];25(6):491-98. Disponível em: http://www.scielosp.org/scielo.php?pid=S102049892009000600004\&script =sci_arttext

4. Sociedade Brasileira de Hipertensão. VI Diretrizes Brasileiras de Hipertensão Arterial. Rev Bras Hipertens [Internet]. 2010 [acesso em 05 de julho de 2014];17(1):4-60. Disponível em: http://www.anad.org.br/profissionais/images/VI_Diretrizes_Bras_Hipertens_RDHA_6485.pdf

5. Ministério da Saúde (BR). Prevenção clínica de doença cardiovascular, cerebrovascular e renal crônica [Internet]. Brasília (DF): Ministério da Saúde; 2006 [acesso em 05 de julho de 2014]. Disponível em: http://189.28.128.100/ dab/docs/publicacoes/cadernos_ab/abcad14.pdf

6. Ministério da Saúde (BR). Estratégias para o cuidado da pessoa com doença crônica: hipertensão arterial sistêmica [Internet]. Brasília (DF): Ministério da Saúde; 2013 [acesso em 05 de julho de 2014]. Disponível em: http:// bvsms.saude.gov.br/bvs/publicacoes/estrategias_cuidado_pessoa_doenca_cronica.pdf

7. Sociedade Brasileira de Cardiologia. IV Diretriz Brasileira sobre Dislipidemias e Prevenção da Aterosclerose: departamento de Aterosclerose da Sociedade Brasileira de Cardiologia. Arq Bras Cardiol. 2007;88(1):2-19.

8. Lemos DM, Fundão LN, Ferreira MVL, Mill JG, Molina MDCB. Redução quantitativa do risco cardiovascular no tratamento da hipertensão arterial em unidade do Programa de Saúde da Família. Rev Bras Hipertens [Internet]. 2006 [acesso em 05 de julho de 2014];13(2):117-25.
Disponívelem:http://departamentos.cardiol.br/dha/revista /13-2/08-reducao-quantitativa.pdf

9. Oliveira EA, Bubach S, Flegeler DS. Perfil de hipertensos em uma unidade de saúde da família. Rev Enferm UERJ [Internet]. 2009 [acesso em 05 de julho de 2014];17(3):383-7. Disponível em: http://www.facenf. uerj.br/v17n3/v17n3a15.pdf

10. Dosse C, Cesarino CB, Martin JFV, Castedo MCA. Fatores associados à não adesão dos pacientes ao tratamento de hipertensão arterial. Rev Latinoam Enferm. 2009;17(2): 201-6.

11. Demoner MS, Ramos ERP, Pereira ER. Fatores associados à adesão ao tratamento anti-hipertensivo em unidade básica de saúde. Acta Paul Enferm [Internet]. 2012 [acesso em 03 de julho de 2014];25(n. ${ }^{\circ}$ esp.1):27-34. Disponível em: http://www.scielo.br/pdf/ape/v25nspe1/pt_05.pdf

12. Carvalho ALM, Leopoldino RWD, Silva JEG, Cunha CP. Adesão ao tratamento medicamentoso em usuários cadastrados no Programa Hiperdia no município de Teresina (PI). Ciênc Saúde Coletiva. 2012;17(7):1885-92.

13. Waidman MAP, Radovanovic CAT, Estevam MC, Marcon SS. Assistência à pessoa com hipertensão arterial na ótica do profissional de saúde. Rev Bras Enferm. 2012;65(3):445-53.

14. Moura DJM, Bezerra STF, Moreira TMM, Fialho AVM. Cuidado de enfermagem ao cliente com hipertensão: uma revisão bibliográfica. Rev Bras Enferm. 2011;64(4):759-65. 